\title{
Potential Use of Rum Distillery Slops as Animal Feed Supplement. III. Effect of pH, Composition and Dilution in Mold Growth and BOD Reduction of Slops ${ }^{1}$
}

\author{
Isabel M. González and Nivia F. Murphy²
}

\begin{abstract}
The growth of different strains of Aspergilli used in this study was affected by changes in the $\mathrm{pH}$ of the slops. Best results were obtained at $\mathrm{pH} 4.8$ or higher. The slops from two distilleries differed in composition, but this did not affect mold growth and did not alter consequent reductions of BOD and total sugars in the treated product. BOD reductions in diluted slops were higher $(75 \%)$ than in undiluted slops $(56 \%)$, increasing with slops dilution. Best results were obtained with a slops water dilution of 1:2.
\end{abstract}

\section{INTRODUCTION}

The use of members of the class Fungi imperfecti as sources of protein food was investigated by Gray et al. (4). These researchers noted that the composition of fungal mycelia was as satisfactory nutritionally as casein, that is, as a source of essential amino acids with a high caloric value. Gray asserted (5) that the growth of fungi on agricultural wastes is a partial solution to the world food problems.

Fungi, like other organisms, have their own specific requirements for optimal growth. Studies performed by Araujo et al. (1) show that strains of Aspergilli are not influenced by changes in $\mathrm{pH}$, temperature, nutrients and aeration.

The Rum Pilot Plant has investigated the fermentation of rum distillery slops with molds to enrich the protein content for use as animal feed supplement (2). The slops proved to be valuable for the growth of mold, with a maximum yield of $17 \mathrm{~g} / \mathrm{L}$ after 4 days' growth in laboratory scale experiments. In another study (3) high reductions of Biological Oxygen Demand (BOD) and total sugars of slops were obtained through mold growth for the production of mycelial protein. These findings should help the Puerto Rican rum industry dispose properly of its effluents.

The present work reports the effect of variations in the substrate's $\mathrm{pH}$, composition and dilution on mold growth and on BOD reduction of rum distillery slops.

\footnotetext{
${ }^{1}$ Manuscript submitted to Editorial Board November 3, 1978.

${ }^{2}$ Associate Chemist and Bacteriologist, respectively, Agricultural Experiment Station, Mayagüez Campus, University of Puerto Rico, Río Piedras, P.R.
} 


\section{MATERIALS AND METHODS}

To determine the optimum pH level of slops for high mycelium and protein yields in a shorter time, we studied mold strain H-13, Aspergillus phoenicis, following the procedure previously described $(2,3)$. Values of $\mathrm{pH}$ from 3.0 to 7.0 were selected for the first test. Mycelium was harvested at 24 and 48 hours. Another test was conducted in which, after 24 hours of growth, the $\mathrm{pH}$ was readjusted to the initial value under study, and then harvested after the 48-hour growth period was completed. Mycelial yield and protein content of samples were determined in both cases.

Following this procedure, we conducted a comparative growth experiment with three mold strains at two different $\mathrm{pH}$ values. Strains H-13,

TABLE 1.-Mycelial production of mold $H-13$ at different $p H$ values

\begin{tabular}{cccccc}
\hline \multirow{2}{*}{ Harvest time } & \multicolumn{5}{c}{ Mycelium production (g/1) } \\
\cline { 2 - 6 } & $3.0 \mathrm{pH}$ & $4.0 \mathrm{pH}$ & $4.8 \mathrm{pH}$ & $6.0 \mathrm{pH}$ & $7.0 \mathrm{pH}$ \\
\hline$H$ & 1.0 & 0.8 & 0.5 & 2.2 & 5.9 \\
24 & 0.8 & 0.6 & 2.5 & 9.1 & 9.6 \\
48 & 0.8 & 0.8 & 4.4 & 10.9 & 9.1 \\
$48^{* 1}$ & &
\end{tabular}

' $\mathrm{pH}$ adjusted at $24 \mathrm{hr}$ to initial $\mathrm{pH}$ under study.

TABLE 2.-Protein content (\%) of mycelia produced by mold H-13 at different $p H$ values

\begin{tabular}{crrrrr}
\hline \multirow{2}{*}{ Harvest time } & \multicolumn{5}{c}{ \% Protein } \\
\cline { 2 - 6 } & $3.0 \mathrm{pH}$ & $4.0 \mathrm{pH}$ & $4.8 \mathrm{pH}$ & $6.0 \mathrm{pH}$ & $7.0 \mathrm{pH}$ \\
\hline$H$ & & & & & \\
24 & 11.25 & 13.75 & 16.25 & 28.75 & 27.50 \\
48 & 11.88 & 15.00 & 23.75 & 27.50 & 25.62 \\
$48^{* 1}$ & 11.88 & 16.25 & 27.50 & 25.62 & 24.38 \\
\hline
\end{tabular}

${ }^{1} \mathrm{pH}$ adjusted at $24 \mathrm{~h}$ to initial $\mathrm{pH}$ under study.

Aspergillus phoenicis; H-21, A. oryzae; and H-23, A. flavus were inoculated from agar slants to liquid synthetic media (6) and after 48 hours of growth, each was transferred to $250 \mathrm{ml}$ sterile slops at $\mathrm{pH}$ values of 4.8 and $6.0 . \mathrm{NaOH}(7 \mathrm{~N})$ was used to increase the $\mathrm{pH}$ of the sterile slops from 4.8 to 6.0. The samples were harvested at 72-hour and 6-day growths. Mycelium was separated by filtration and dried for mycelium yield and protein content. The supernatant and the initial samples were analyzed for $\mathrm{pH}$, protein, $\mathrm{BOD}$, and total sugars.

We determined the effect of composition of substrate, using slops from two distilleries: D-1, Bacardí; and D-2, P.R. Distillers. Mold strain H-13 was grown with mechanical agitation in triplicate samples of sterile slops from both distilleries. Samples were harvested after 5 and 8 days' growth. 
Mycelium was separated by filtration and the supernatant was analyzed for $\mathrm{BOD}, \mathrm{pH}$, and total sugars. These analyses were also performed before and after sterilizing the slops.

Growth of molds in diluted slops and the effect on reduction of BOD and total sugars were studied. A first experiment was conducted with Aspergillus flavus, $\mathrm{H}-23$ in slops diluted 1:1 with tap water, sea water and distilled water. Triplicate samples of each sterile diluted medium

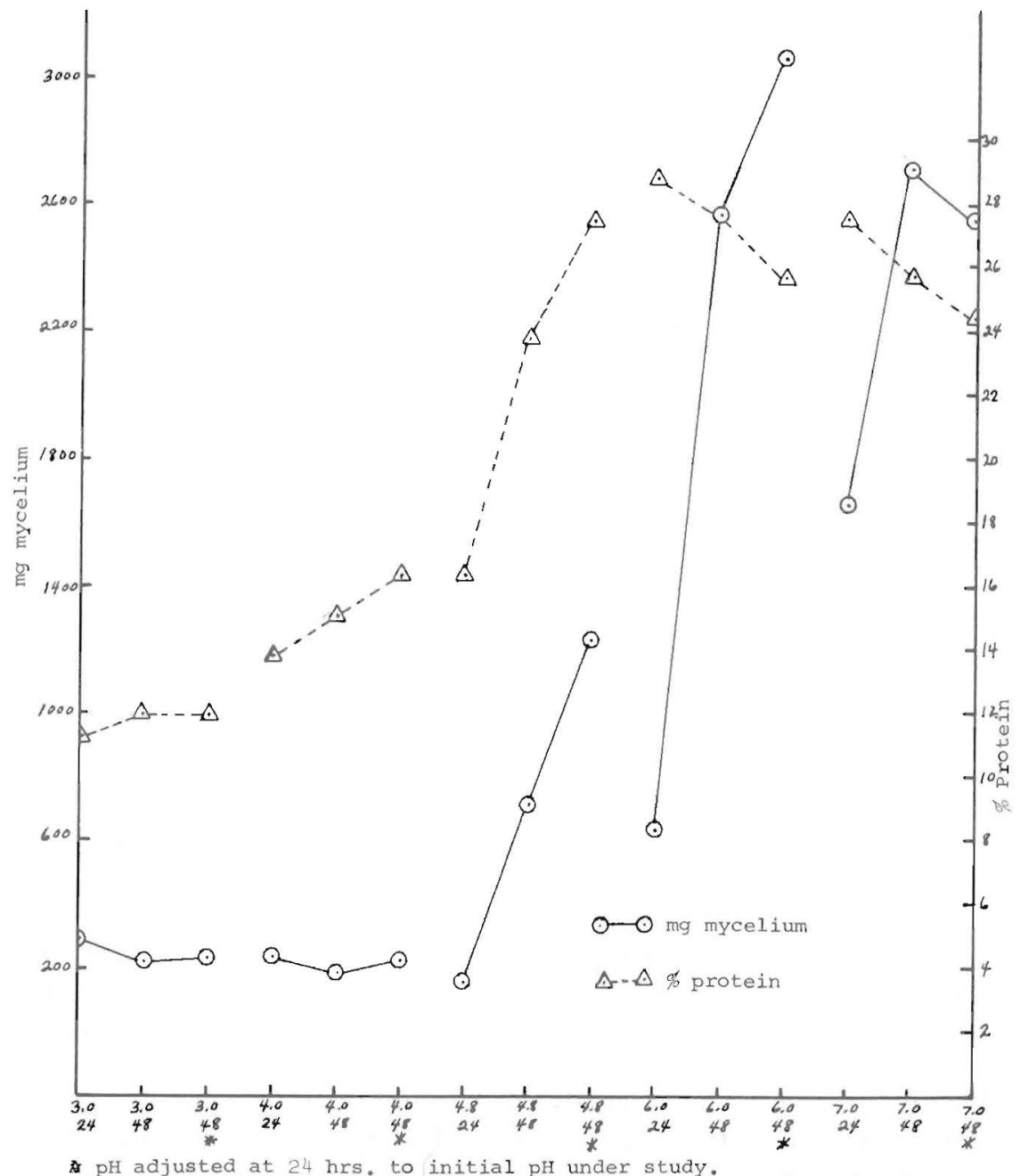

FIG. 1.-Mycelium and protein produced in slops at different $\mathrm{pH}$ values. 
TABLE 3.-Growth of three mold strains at different $p H$ values

\begin{tabular}{|c|c|c|c|c|c|c|c|c|c|c|c|}
\hline \multirow{2}{*}{ Sample } & \multirow{2}{*}{$\begin{array}{l}\text { Mold } \\
\text { strain }\end{array}$} & \multicolumn{2}{|c|}{$\mathrm{pH}$ values } & \multirow{2}{*}{$\begin{array}{c}\text { Harvest } \\
\text { time }\end{array}$} & \multirow{2}{*}{$\begin{array}{c}\text { Mycelium } \\
\text { weight }\end{array}$} & \multirow{2}{*}{$\begin{array}{c}\text { Mycelium } \\
\text { protein }\end{array}$} & \multirow{2}{*}{ BOD } & \multirow{2}{*}{$\begin{array}{l}\text { Reduction } \\
\text { BOD }\end{array}$} & \multirow{2}{*}{$\begin{array}{l}\text { Total } \\
\text { sugar }\end{array}$} & \multirow{2}{*}{$\begin{array}{l}\text { Reduction } \\
\text { sugar }\end{array}$} & \multirow{2}{*}{$\begin{array}{c}\text { Supernatant } \\
\text { protein }\end{array}$} \\
\hline & & Initial & Final & & & & & & & & \\
\hline & & & & & $G / L$ & $\%$ & $p / m$ & $\%$ & $\mathrm{~g} / 100 \mathrm{ml}$ & $\%$ & $\mathrm{mg} / 100 \mathrm{ml}$ \\
\hline A-11 & - & 4.8 & - & - & - & - & 42,500 & - & 1.60 & - & 1149 \\
\hline A-12 & H-13 & 4.8 & 5.3 & 72 hrs. & 13.0 & 22.68 & 35,700 & 16 & 0.65 & 59 & 794 \\
\hline A-13 & H-13 & 4.8 & 5.3 & 6 days & 15.8 & 18.61 & 25,250 & 40 & 0.39 & 76 & 898 \\
\hline A-14 & H-13 & 6.0 & 5.6 & 72 hrs. & 8.8 & 22.31 & 34,850 & 18 & 0.55 & 66 & 859 \\
\hline A-15 & H-13 & 6.0 & 7.4 & 6 days & 9.8 & 16.74 & 24,000 & 43 & 0.41 & 74 & 982 \\
\hline A-16 & H-21 & 4.8 & 5.6 & $72 \mathrm{hrs}$ & 10.9 & 26.71 & 35,700 & 16 & 0.50 & 69 & 784 \\
\hline A- 17 & H-21 & 4.8 & 5.9 & 6 days & 15.1 & 23.19 & 23,250 & 45 & 0.38 & 76 & 796 \\
\hline A-18 & H-21 & 6.0 & 5.7 & 72 hrs. & 9.8 & 25.87 & 36,550 & 14 & 0.39 & 76 & 825 \\
\hline A- 19 & $\mathrm{H}-21$ & 6.0 & 5.5 & 6 days & 8.4 & 19.30 & 29,500 & 30 & 0.35 & 78 & 1003 \\
\hline A-20 & $\mathrm{H}-23$ & 4.8 & 5.2 & 72 hrs. & 13.7 & 26.27 & 34,850 & 18 & 0.48 & 70 & 763 \\
\hline A-21 & $\mathrm{H}-23$ & 4.8 & 6.3 & 6 days & 16.8 & 21.21 & 24,000 & 44 & 0.35 & 78 & 784 \\
\hline A-22 & $\mathrm{H}-23$ & 6.0 & 5.9 & 72 hrs. & 10.9 & 28.03 & 32,300 & 24 & 0.37 & 76 & 857 \\
\hline A-23 & H-23 & 6.0 & 5.5 & 6 days & 8.8 & 19.23 & - & - & 0.33 & 79 & 974 \\
\hline
\end{tabular}


were inoculated and mechanically agitated for 6 days. Sterile undiluted slops were equally inoculated as control. The treated and untreated samples were analyzed for ${ }^{\circ}$ Brix, $\mathrm{pH}, \mathrm{BOD}$ and total sugars. Mycelium was separated by filtration to determine the yield and nitrogen content.

Following the previously mentioned procedure, a second experiment was conducted with various dilutions of slops:tap water, using mold strain H-23, Aspergillus flavus. Five dilutions were compared: 1:0, 1:1, 1:2, 2:1, and 3:1. Duplicate samples were inoculated and harvested after 5 days' growth with mechanical agitation. Initial and final (supernatant) samples were analyzed for total organic carbon (TOC), ${ }^{\circ} \mathrm{Brix}, \mathrm{pH}, \mathrm{BOD}$, total

TABLE 4.-BOD and sugar analysis at various conditions

\begin{tabular}{|c|c|c|c|c|c|c|c|c|}
\hline \multirow{2}{*}{ Sample } & \multirow{2}{*}{$\begin{array}{c}\text { Sample } \\
\text { description }\end{array}$} & \multirow{2}{*}{$\begin{array}{l}\text { Slops } \\
\text { source }\end{array}$} & \multirow{2}{*}{$\mathrm{BOD}$} & \multirow{2}{*}{$\begin{array}{l}\text { Reduction } \\
\text { BOD }\end{array}$} & \multirow{2}{*}{$\begin{array}{l}\text { Total } \\
\text { sugar }\end{array}$} & \multirow{2}{*}{$\begin{array}{l}\text { Reduction } \\
\text { sugar }\end{array}$} & \multicolumn{2}{|c|}{$\mathrm{pH}$} \\
\hline & & & & & & & Initial & Final \\
\hline & \multirow{4}{*}{$\begin{array}{l}\text { Before } \\
\text { sterilization }\end{array}$} & & $p / m$ & $\%$ & $\mathrm{~g} / 100 \mathrm{ml}$ & $\%$ & & \\
\hline a & & D-1 $1^{\prime}$ & 36,250 & - & 1.48 & - & 4.8 & - \\
\hline b & & D-1 & 40,000 & - & 1.46 & - & 4.8 & - \\
\hline$c$ & & D-1 & 39,250 & - & 1.47 & - & 4.8 & - \\
\hline$d$ & \multirow{3}{*}{$\begin{array}{l}\text { Before } \\
\text { sterilization }\end{array}$} & D- $2^{2}$ & 35,250 & - & 0.90 & - & $4: 4$ & - \\
\hline e & & D-2 & 35,000 & - & 0.91 & - & 4.4 & - \\
\hline f & & D-2 & 33,000 & - & 0.93 & - & 4.4 & - \\
\hline $\mathrm{A}-24$ & \multirow[t]{3}{*}{ After sterilization } & D-1 & 54,000 & - & 1.33 & - & 4.8 & - \\
\hline A-25 & & D-1 & 54,000 & - & 1.45 & - & 4.8 & - \\
\hline A- 26 & & D-1 & 47,000 & - & 1.44 & - & 4.8 & - \\
\hline $\mathrm{A}-27$ & \multirow[t]{3}{*}{ After sterilization } & D-2 & 43,000 & - & 0.91 & - & 4.4 & - \\
\hline A-28 & & D-2 & 41,000 & - & 0.91 & - & 4.4 & - \\
\hline A-29 & & D-2 & 36,000 & - & 0.95 & - & 4.4 & - \\
\hline A-30 & \multirow{3}{*}{$\begin{array}{l}\text { Mold growth har- } \\
\text { vested at } 5 \text { days }\end{array}$} & D-1 & 25,400 & 51 & 0.70 & 52 & 4.8 & - \\
\hline A-31 & & D-1 & 25,025 & 52 & 0.76 & 48 & 4.8 & - \\
\hline A-32 & & D-1 & 26,650 & 49 & 0.75 & 49 & 4.8 & - \\
\hline A-33 & \multirow{3}{*}{$\begin{array}{l}\text { Mold growth har- } \\
\text { vested at } 5 \text { days }\end{array}$} & D-2 & 20,275 & 49 & 0.44 & 52 & 4.4 & - \\
\hline A-34 & & D-2 & 22,525 & 44 & 0.67 & 27 & 4.4 & - \\
\hline A-35 & & D-2 & 20,925 & 48 & 0.57 & 38 & 4.4 & - \\
\hline A-36 & \multirow{3}{*}{$\begin{array}{l}\text { Mold growth har- } \\
\text { vested at } 8 \text { days }\end{array}$} & D-1 & 25,000 & 52 & 0.78 & 47 & 4.8 & 4.8 \\
\hline A-37 & & D-1 & 24,000 & 54 & 0.78 & 47 & 4.8 & 4.9 \\
\hline A-38 & & D-1 & 23,600 & 55 & 0.60 & 59 & 4.8 & 5.1 \\
\hline A-39 & \multirow{3}{*}{$\begin{array}{l}\text { Mold growth har- } \\
\text { vested at } 8 \text { days }\end{array}$} & D-2 & 21,000 & 47 & 0.59 & 36 & 4.4 & 4.5 \\
\hline A-40 & & D-2 & 20,700 & 48 & 0.57 & 38 & 4.4 & 4.5 \\
\hline$A-41$ & & D-2 & 20,100 & 50 & 0.50 & 46 & 4.4 & 4.5 \\
\hline
\end{tabular}

${ }^{1} \mathrm{D}-1=$ Bacardi slops.

${ }^{2} \mathrm{D}-2=\mathrm{P}$. R. Distillers slops. 
sugars and protein percentage. Mycelium was harvested by filtration and dried for yield and protein determinations.

\section{RESULTS AND DISCUSSION}

Tables 1 and 2 show the data on the influence of various $\mathrm{pH}$ values on the mold H-13 growth. These results are also shown graphically in figure 1. Best mycelial and protein yields were obtained at $\mathrm{pH}$ 6.0.

Table 3 shows comparative growth data of three mold strains at two different initial pH values. Inconsistency was observed in terms of my-

TABLE 5.-Mold growth in diluted slops

\begin{tabular}{ccccccccccc}
\hline $\begin{array}{c}\text { Sam- } \\
\text { ple }\end{array}$ & $\begin{array}{c}\text { Dilution } \\
\text { slops: Wa- } \\
\text { ter }\end{array}$ & $\begin{array}{c}\text { Myce- } \\
\text { lium } \\
\text { weight }\end{array}$ & ${ }^{\circ} \mathrm{Bx}$ & $\mathrm{pH}$ & $\mathrm{BOD}$ & $\begin{array}{c}\text { Reduc- } \\
\text { tion } \\
\mathrm{BOD}\end{array}$ & $\begin{array}{c}\text { Total } \\
\text { sugar }\end{array}$ & $\begin{array}{c}\text { Total } \\
\text { Reduc- } \\
\text { tion } \\
\text { sugar }\end{array}$ & $\begin{array}{c}\text { Myce- } \\
\text { lium } \\
\text { protein }\end{array}$ & $\begin{array}{c}\text { Superna- } \\
\text { tant } \\
\text { protein' }\end{array}$ \\
\hline & $\mathrm{g} / l$ & & & $p / m$ & $\%$ & $\begin{array}{c}\mathrm{g} / 100 \\
m l\end{array}$ & $\%$ & $\%$ \\
$\mathrm{~A}-42$ & $1: 0$ Initial & - & 12.8 & 5.8 & 49,200 & - & 1.10 & - & - & 1295 \\
$\mathrm{~A}-43$ & $1: 0$ & 22.5 & 10.3 & 6.4 & 18,125 & 55 & 0.50 & 55 & 24.14 & 836 \\
A-44 & $1: 0$ & 24.0 & 10.3 & 6.7 & 17,125 & 57 & 0.48 & 56 & 23.22 & 773
\end{tabular}

Tap water

$\begin{array}{lllllllllll}\text { A-45 } & 1: 1 \text { Initial } & - & 6.8 & 5.2 & 20,450 & - & 0.43 & - & - & 648 \\ \text { A-46 } & 1: 1 & 14.7 & 5.0 & 8.2 & 4,250 & 79 & <0.03 & >93 & 21.51 & 355 \\ \text { A-47 } & 1: 1 & 13.7 & 5.0 & 8.0 & 5,550 & 73 & <0.03 & >93 & 24.06 & 355\end{array}$

Sea water

$\begin{array}{llllllllllr}\text { A-48 } & 1: 1 \text { Initial } & - & 9.9 & 5.0 & 20,200 & - & 0.44 & - & - & 648 \\ \text { A-49 } & 1: 1 & 13.6 & 8.0 & 7.8 & 5,550 & 73 & <0.03 & >91 & 21.31 & 0 \\ \text { A-50 } & 1: 1 & 14.3 & 8.2 & 8.0 & 4,650 & 77 & <0.03 & >91 & 26.44 & 125\end{array}$

Distilled water

\begin{tabular}{llllllllllr}
$\mathrm{A}-51$ & $1: 1$ Initial & - & 6.8 & 5.1 & 20,050 & - & 0.44 & - & - & 648 \\
$\mathrm{~A}-52$ & $1: 1$ & 14.7 & 5.0 & 8.2 & 3,750 & 81 & $<0.03$ & $>91$ & 21.34 & 0 \\
$\mathrm{~A}-53$ & $1: 1$ & 14.5 & 4.9 & 8.0 & 6,750 & 66 & $<0.03$ & $>91$ & 21.44 & 0 \\
\hline
\end{tabular}

' mg/100 ml. Mold strain H-23.

celium protein produced at pH 4.8 vs. 6.0, but mycelium yield was higher at $\mathrm{pH} 4.8$ than at $\mathrm{pH}$ 6.0. This result differed from previous findings. As growth progresses, mycelium protein decreases while supernatant protein increases, suggesting mycelium autolysis. Variations in BOD and total sugar reductions at $\mathrm{pH} 4.8$ vs. 6.0 were not significant.

Table 4 summarizes results on mold growth in slops from different origins. BOD increased significantly after sterilization, while sugar content remained constant. This indicates that the increase in BOD could not be attributed to slops concentration during the sterilization process. Slops from two distilleries were different in composition, but this did not 
affect mold growth and subsequent BOD and total sugar reductions. Harvesting after an 8-day growth instead of a 5-day growth did not make any difference in the tendency of $\mathrm{BOD}$ and total sugar reductions.

Table 5 presents results on the effect of slops dilution in mold growth and $\mathrm{BOD}$ reductions. These are shown graphically in figure 2. BOD and total sugar reductions in diluted slops 1:1 were higher $(75 \%)$ than in undiluted slops (56\%). The yield of mycelia obtained in diluted samples

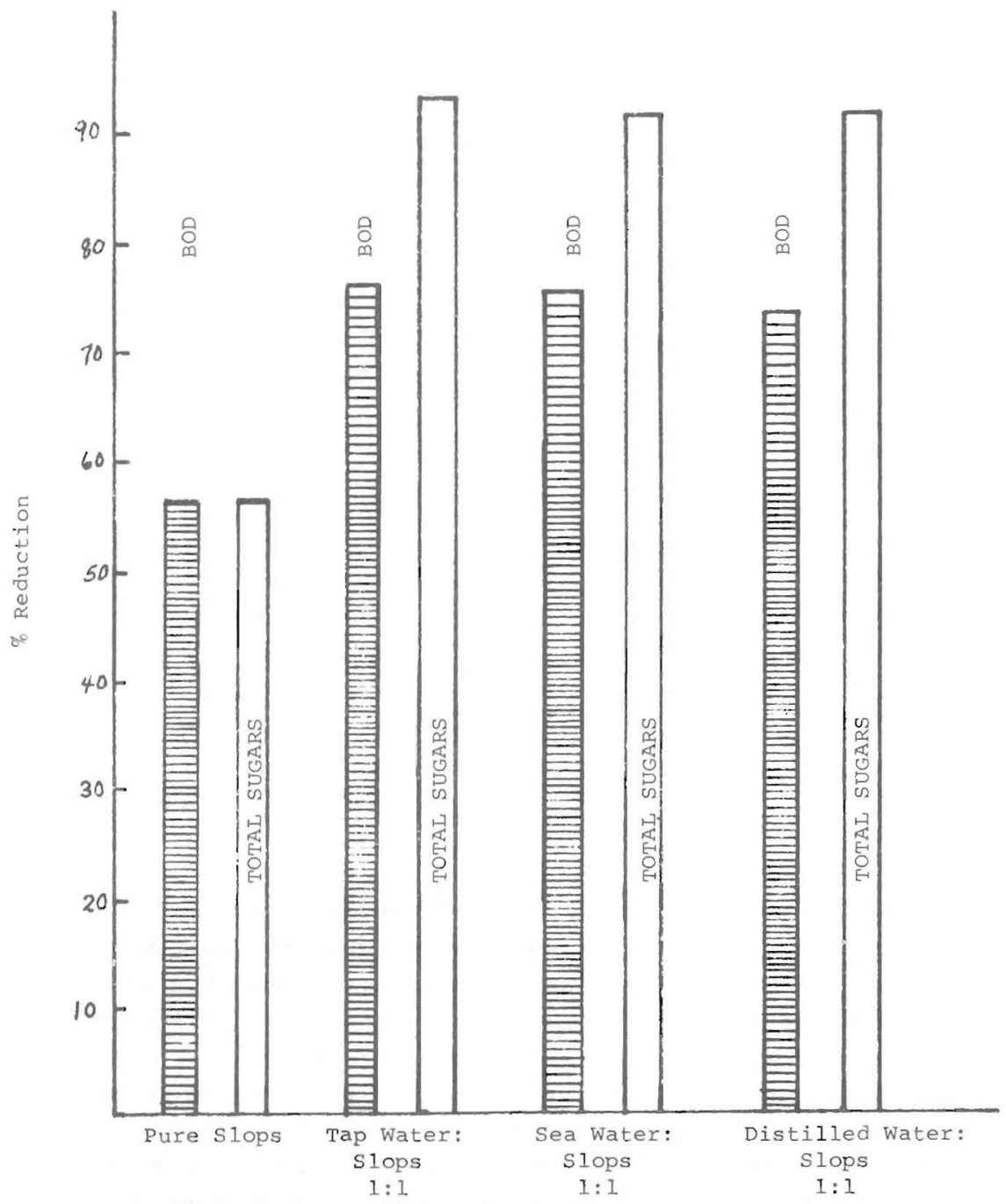

FIG. 2.-Effect of slops dilution in BOD and sugar reductions during mold growth 
TABLE 6.-Effect of slops dilution in BOD and sugar reductions during mold growth

\begin{tabular}{|c|c|c|c|c|c|c|c|c|c|c|c|}
\hline $\begin{array}{l}\text { Dilution } \\
\text { slops: water }\end{array}$ & $\mathrm{pH}$ & ${ }^{\circ} \mathrm{BX}$ & $\begin{array}{l}\text { Total } \\
\text { sugar }\end{array}$ & $\begin{array}{l}\text { Total } \\
\text { reduction } \\
\text { sugar }\end{array}$ & $\mathrm{BOD}$ & $\begin{array}{l}\text { BOD } \\
\text { reduction }\end{array}$ & TOC & $\begin{array}{l}\text { TOC } \\
\text { reduction }\end{array}$ & $\begin{array}{c}\text { Mycelium } \\
\text { weight }\end{array}$ & $\begin{array}{l}\text { Mycelium } \\
\text { protein }\end{array}$ & $\begin{array}{l}\text { Super- } \\
\text { natant } \\
\text { protein }\end{array}$ \\
\hline & & & $\mathrm{g} / 100 \mathrm{ml}$ & $\%$ & $p / m$ & $\%$ & $p / m$ & $\%$ & $g / l$ & $\%$ & \\
\hline 1:0 Initial & 4.9 & 13.3 & 1.16 & - & 38,000 & - & 45,500 & - & - & - & 1,120 \\
\hline 1:0 & 6.0 & 10.8 & 0.62 & 46 & 22,250 & 41 & 28,750 & 37 & 17.3 & 24.77 & 773 \\
\hline 1:0 & 6.1 & 10.9 & 0.57 & 51 & 22,250 & 41 & 35,750 & 21 & 17.2 & 25.73 & 752 \\
\hline 3:1 Initial & 4.9 & 10.1 & 0.85 & - & 23,250 & - & 29,000 & - & - & - & 840 \\
\hline $3: 1$ & 6.7 & 7.9 & 0.30 & 65 & 13,300 & 42 & 21,250 & 27 & 15.5 & 24.47 & 499 \\
\hline $3: 1$ & 6.8 & 7.7 & 0.28 & 67 & 11,650 & 50 & 23,000 & 21 & - & - & 439 \\
\hline 2:1 Initial & 4.9 & 8.6 & 0.70 & - & 20,500 & - & 27,000 & - & - & - & 746 \\
\hline $2: 1$ & 7.1 & 6.9 & 0.15 & 79 & 9,050 & 56 & 20,500 & 24 & 15.0 & 23.83 & 418 \\
\hline $2: 1$ & 7.5 & 7.0 & 0.20 & 71 & 9,150 & 55 & 19,250 & 29 & 15.5 & 22.68 & 125 \\
\hline 1:1 Initial & 5.1 & 6.4 & 0.46 & - & 16,250 & - & 25,600 & - & - & - & 560 \\
\hline $1: 1$ & 7.7 & 5.4 & 0.07 & 83 & 7,700 & 53 & 15,500 & 39 & 12.5 & 23.60 & 230 \\
\hline $1: 1$ & 7.7 & 5.4 & 0.05 & 85 & 6,450 & 60 & 15,340 & 40 & 12.4 & 22.47 & 276 \\
\hline 1:2 Initial & 5.0 & 4.2 & 0.19 & - & 11,400 & - & 10,820 & - & - & - & 373 \\
\hline $1: 2$ & 7.9 & 3.7 & -0.03 & +84 & 4,075 & 64 & 8,320 & 23 & 9.7 & 23.26 & 165 \\
\hline $1: 2$ & 7.9 & 3.6 & -0.03 & +84 & 3,625 & 68 & 8,400 & 22 & 9.5 & 22.89 & 207 \\
\hline
\end{tabular}

${ }^{1} \mathrm{mg} / 100 \mathrm{ml}$. Mold strain H-23. 
was $62 \%$ of the undiluted samples; we expected $50 \%$. An average of 14.2 $\mathrm{g} / \mathrm{L}$ of mycelium was obtained with an average protein content of $22.7 \%$ from diluted samples. Undiluted samples produced $23.2 \mathrm{~g} / \mathrm{L}$ with $23.7 \%$ protein content. No significant variations were observed when different types of water were used for dilution.

Table 6 and figure 3 show that with the more dilute slops, there was

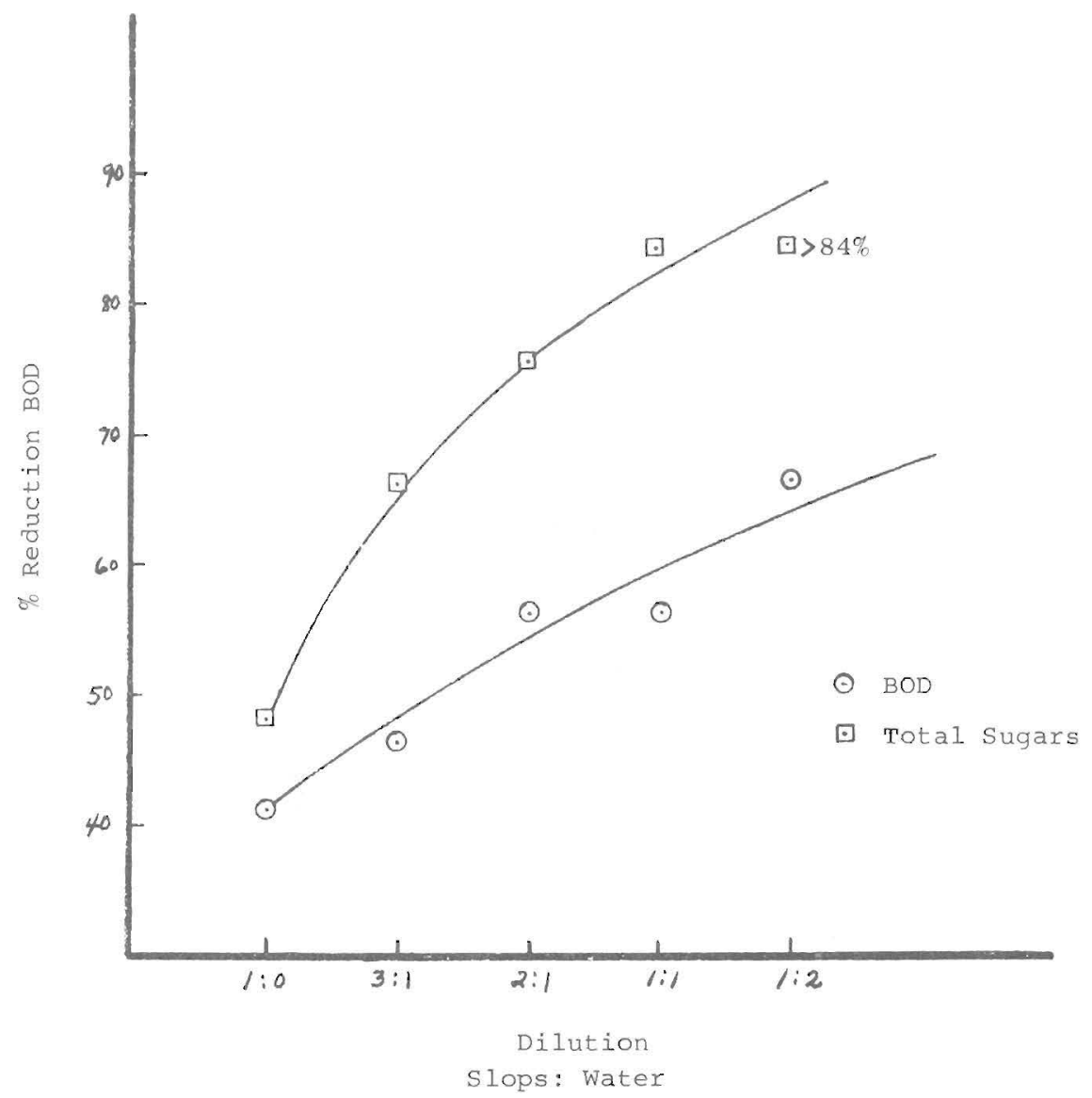

Frg. 3.-Effect of slops dilution in $\mathrm{BOD}$ and sugar reductions during mold growth

more reduction in $\mathrm{BOD}$ and total sugar content of slops after mold growth. Best results were obtained with a slops:water dilution of 1:2. Protein content of mycelia remained constant. No differences were observed in the total organic carbon (TOC) after mold growth.

\section{RESUMEN}

La técnica para el crecimiento de varias especies de hongos del género aspergilo en mostos ${ }^{3}$ demostró que éstos crecen mejor en pH 
4.8 o más alto. La variación en la composición de los mostos obtenidos de dos destilerías de ron no afectó el crecimiento de los hongos ni las reducciones en BOD y su contenido en azúcar total. La dilución de los mostos para cultivar hongos aumentó significativamente las reducciones en BOD de $56 \%$ sin diluir a $75 \%$ diluido. Los mejores resultados se obtuvieron con una dilución de mostos en agua de 1:2.

${ }^{3}$ Vinaza; residuo de la fermentación y destilación de la melaza de la caña de azúcar.

\section{LITERATURE CITED}

1. Araujo, N. de Q., Visconti, A. S., de Castro, H. F., da Silva, H. G. B., Ferraz, M. H. A., and Filho, M. S., 1976. Production of fungal biomass from vinasse, Bras. Açucarer. 88, 479-89 (Portuguese).

2. González, I. M. and Murphy, N. F., 1979. Potential use of rum distillery slops as animal feed supplement. I. Mold growth in slops, J. Agri. Univ, P. R. 63(3): 325-9

3. - and - 1979. Potential use of rum distillery slops as animal feed supplement. II. Nitrogen content of mycelial growth in slops, J. Agri. Univ. P. R. 63 (3):330-5.

4. Gray, W. D., Pinto Patrick, V. C., and Pathak, S. G., 1963. Growth of fungi in sea water medium, Appl. Microbiol. 11, 501.

5. - - 1966. Fungi and world protein supply, Adv. Chem. Ser. 57, 261-8.

6. Personal Técnico de la Planta Piloto de Ron, 1969. Manual de métodos y procedimientos bacteriológicos de la Planta Piloto de Ron, Universidad de Puerto Rico, Recinto Universitario de Mayagüez, Esta. Exp. Agrí., Univ. P.R. 